\title{
SEGMENTATION OF RETINAL ARTERIAL BIFURCATIONS IN 2D ADAPTIVE OPTICS OPHTHALMOSCOPY IMAGES
}

\author{
Iyed Trimeche ${ }^{1,2,3}$, Florence Rossant ${ }^{1}$, Isabelle Bloch ${ }^{2}$, Michel Paques ${ }^{3}$ \\ 1. Institut Supérieur d'Electronique de Paris (ISEP), Paris, France \\ 2. LTCI, Télécom ParisTech, Université Paris-Saclay, Paris, France \\ 3. Clinical Investigation Center 1423, Quinze-Vingts Hospital, Paris, France \\ iyed.trimeche@isep.fr
}

\begin{abstract}
The study of vascular morphometry requires segmenting vessels with high precision. Of particular clinical interest is the morphometric analysis of arterial bifurcations in Adaptive Optics Ophthalmoscopy (AOO) images of eye fundus. In this paper, we extend our previous approach for segmenting retinal vessel branches to the segmentation of bifurcations. This enables us to recover the microvascular tree and extract biomarkers that charactarize the blood flow. Segmentation results are shown to be within the range of intraand inter-user variability, allowing a preliminary study on biomarkers derived from vessel diameter estimates at arterial bifurcations.
\end{abstract}

Index Terms - Active contours, segmentation, retinal arterial bifurcations, adaptive optics ophthalmoscopy images.

\section{INTRODUCTION}

This study aims to determine the effect of CADASIL syndrome on vessel morphometry, by segmenting retinal vessels precisely in Adaptive Optics Ophthalmoscopy (AOO) and then derive some biomarkers. This rare pathology is an inherited condition that causes stroke and other impairments affecting blood flow in small blood vessels, particularly vessels within the brain [1]. Retinal vessels are related to cerebral vessels, sharing many structural, functional, and pathological features. Therefore, retinal vessels may be considered in many ways as substitutes for the cerebral vessels. Moreover, they are more easily observable thanks to their planar arrangement and to dedicated high resolution imaging systems, such as AOO (Fig. 1). For all these reasons, we can assume that the analysis of retinal vessel alterations observable in $2 \mathrm{D}$ AOO images will enable us to define relevant biomarkers for CADASIL syndrome.

In addition to the general considerations of contrast, resolution, noise and acquisition dependent artifacts, retinal vascular networks are complex tree-like structures with successive branchings. To analyze these structures, the relationships between the lumens (i.e. diameters) of bifurcating blood vessels are shown to arise from some simple principles of optimality, as already suggested by D'Arcy Thompson [2]. The best known of these is Murray's law [3]. This law led to the definition of many biomarkers that describe the relationship between parent vessel and child branches in a bifurcation. Measuring diameter dependent biomarkers on patients has shown deviations from Murray's optimality, related with some pathologies such as stroke [4], diabetes [5] and high blood pressure [6]. These

This research has been partially funded by a grant from the French Agence Nationale de la Recherche ANR-16-RHUS-0004 (RHU TRT cSVD). deviations can be related to blood circulation disorders and reflect the progress of some pathologies.

The estimation of retinal artery lumen at a bifurcation requires an accurate segmentation of the vascular tree, which is a very difficult but also valuable task especially for studying vessel morphometry and for diagnosis assistance [7]. In fact, most clinical studies rely on standard eye fundus images (the interior wall of the eye) [4, 6]. Limits of the proposed protocols come from the poor resolution of the images (about 10 to $20 \mu \mathrm{m} / \mathrm{pixel}$ ) and the methods used to estimate the diameters. In [5], such estimations are based on manually defined points, so with limited accuracy and reproducibility. AOO offers a much higher image resolution (about 1 to $2 \mu \mathrm{m} / \mathrm{pixel}$ ), and potentially more accurate biomarker estimates, but, to the best of our knowledge, there does not exist yet a fully automatic and reliable segmentation algorithm to this end.

This paper presents a semi-automatic method for precise segmentation of arterial bifurcations in 2D AOO images, which extends our previous work on segmentation of individual arterial branches [8]. The algorithm segments the branches according to the method proposed in [8], then the segmentation is refined at bifurcations following our new approach. Diameter estimation at bifurcations is then performed, as well as a preliminary study of biomarker extraction on healthy and pathological cases. Finally, we evaluate quantitatively diameters and biomarkers measured from our segmentation.

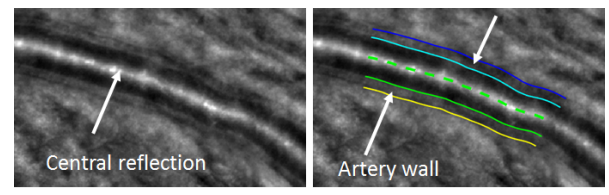

Fig. 1: Segmentation of an AOO image of a retinal artery [8].

\section{STATE OF THE ART}

Conventional fundus photographs were generally used to estimate biomarkers at arterial bifurcation or venous confluences [5, 6]. AOO allows for a better resolution and hence a potentially more accurate estimate of diameters and biomarkers. Arichika et al. [9] segmented AOO images with a semi-automatic method: spline interpolation is applied to determine the limits of the vessel lumen from control points placed manually, and then estimate the diameters. This approach is operator-dependent and lacks reproducibility; it is not tractable when processing large databases. A fully automatic method for segmenting artery walls was developed by Lerme et al. [8], who 
proposed exploiting prior information on the geometry of the vessels and their gray levels in AOO images. This method models the arterial wall as four curves approximately parallel to a common reference line placed on the central reflection (Fig. 1). These curves are initialized automatically through a tracking procedure and this initial segmentation is refined using an active contour model embedding a parallelism constraint [10]. This algorithm, called AOV, was meant to delineate the wall of any artery branch referenced by its central reflection, but does not provide an accurate segmentation at bifurcations. Contours cross at the bifurcation but do not follow the lumen borders (see Fig. 2a). Moreover, the fully automatic localization of central reflections (and so of the vessel branches) is not reliable enough.

Hence, in this article, we propose a new approach to segment accurately the bifurcations. It is based on three steps: (1) a manual step where the user defines the three vessel branches involved in the bifurcation by placing points on the central reflections; (2) the automatic segmentation of the branches by AOV algorithm; (3) the segmentation of the bifurcation, described in Section 3, which is the key contribution of this paper. This way, the tedious manual segmentation task is avoided, as the user's action is limited to an easy initialization step, and the bifurcation segmentation itself is fully automatic. Finally, the diameters are estimated.

\section{SEGMENTATION OF RETINAL BIFURCATIONS}

In this section, we present the proposed method for bifurcation segmentation. Starting from a manual initialization, i.e a set of points manually placed on the central reflection of the three vessel branches, we apply AOV [8] to segment them accurately. Fig. 2a shows the three pairs of curves that delineate the lumen of the vessel branches (the outer borders are not used in this study).
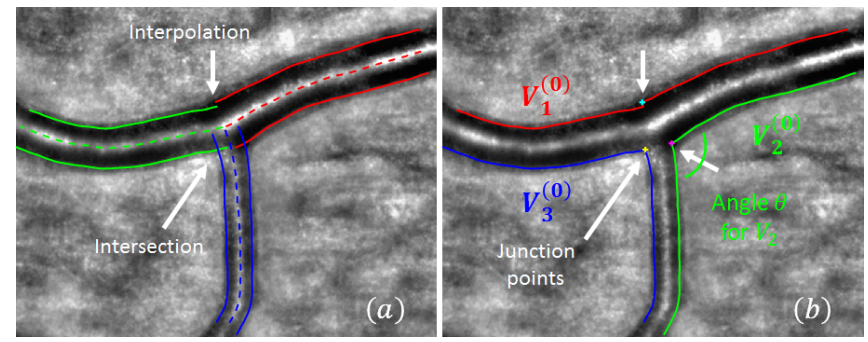

Fig. 2: Initialization of the active contour model. (a) Initial segmentation of vessel branches obtained with AOV semi-automatic mode. (b) Curves $V_{i}^{(0)}$ obtained after rearrangement of the 6 curves delineating the 3 branches involved in the bifurcation.

While this result is generally accurate along the vessel branches, this may not always be the case at the bifurcations. In this study, where diameters have to be measured closed to the bifurcations to derive relevant biomarkers, a precise segmentation is needed even at bifurcations. Therefore we propose to improve the previous result in the following way. We detect the intersection points between two contours (see the crossing contours in Fig. 2a) and we eliminate the rest of the segmentation lines after their crossing. If there is no crossing of segmentation lines, a linear interpolation is applied to reconnect segmentation contours. The resulting curves are rearranged to obtain three segmentation lines, denoted by $V_{i}^{(0)}$, as shown in Fig. 2b. The locations of the former intersection or re-connection points are referred to as junction points and point out regions where the segmentation has to be refined. This new configuration is considered the initialization of an active contour model whose purpose is to ensure that the contours converge precisely to the inner wall of the vessels at the bifurcation without moving out of that region, where the initial segmentation was precise. We propose the following energy functional to be minimized by this deformable model:

$$
E\left(V_{i}\right)=\int_{0}^{1}-\left|\nabla I\left(V_{i}(s)\right)\right|+\alpha(s)\left|V_{i}^{\prime}(s)\right|^{2}+\varphi(s)\left|V_{i}(s)-V_{i}^{(0)}(s)\right|^{2} d s
$$

where $V_{i}$ is one of the three contours, parametrized by $s$, and $V_{i}^{(0)}$ is the corresponding initial contour. In Eq. 1, the first term attracts $V_{i}$ towards the strong gradients of the image, denoted by $I$. The second term is a first regularization for smoothing the curve at the bifurcations, and the last term is a second regularization which insures that the curve $V_{i}$ remains close to the initialization $V_{i}^{(0)}$. The weights $\alpha(s)$ and $\varphi(s)$ are defined according to two criteria, the angle of the re-connected curves at the junction point and the distance to this point. The aim is to impose a strong regularization when moving away from the junction point, to keep the initial position, and to relax the regularization around the junction point, so that the curve can accurately move towards the inner border of the lumen at the bifurcation. This imposes $\varphi(s)$ to be high for points far from the junction point and low around it. Moreover, we also take into account the angles of the reconnected curves at the junction point (Fig. 2b): we need a strong regularization for flat angles, so high values for $\alpha(s)$, and weak values for acute angles, to avoid shortcuts. Thus, to calculate the weight profiles, we rely on a function $f_{p}^{\left(s_{0}, \delta\right)}(s)$ of the curvilinear abscissa $s$, parametrized by the position $s_{0}$ of the junction point and a margin $\delta$ around it (Fig. 3):

$$
f_{p}^{\left(s_{0}, \delta\right)}(s)=\max \left(\frac{1}{1+\exp \left(-\left(s-s_{0}-\delta\right)\right)}, \frac{1}{1+\exp \left(-\left(s_{0}-s-\delta\right)\right)}\right)
$$

The margin $\delta$ is calculated from a rough estimate of the mean diameters $d_{b 1}$ and $d_{b 2}$ of the two branches involved in the processed contour $V_{i}(s)$ :

$$
\delta=\min \left(d_{b 1}, d_{b 2}\right)
$$

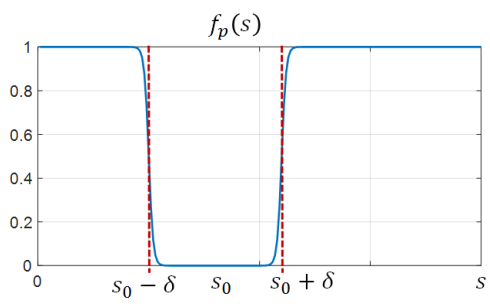

Fig. 3: Function $f_{p}^{\left(s_{0}, \delta\right)}(s)$ parameterized by the curvilinear abscissa $s_{0}$ of the junction point and the margin $\delta$ around it.

Then, the value of $\varphi$ is simply given by:

$$
\varphi(s)=\varphi_{0} f_{p}^{\left(s_{0}, \delta\right)}(s)
$$

with $\varphi_{0}=10$. Thus, the proximity constraint with respect to the initial curve is totally relaxed at the bifurcation, over a distance comparable to the smallest diameter of the two branches involved. It is weighted by $\varphi_{0}$ otherwise.

The second weight $\alpha(s)$ follows a similar profile (Eq. 2, Fig. 3) but it evolves between a maximum value $\alpha_{h i g h}$, set experimentally, and a minimal value $\alpha_{\min }$ calculated from the angle $\theta$ formed by the two initial curves at the junction point (Fig. 2b) :

$$
\alpha_{\text {min }}(\theta)=\alpha_{\text {low }}+\left(\alpha_{\text {high }}-\alpha_{\text {low }}\right) \frac{1}{1+\exp \left(-\gamma\left(\theta-\theta_{\text {med }}\right)\right)}
$$


where $\gamma=0.1, \alpha_{\text {low }}=10, \alpha_{\text {high }}=75$ and $\theta_{\text {med }}=115^{\circ}$. Thus, the value $\alpha_{\min }$ ranges between $\alpha_{\text {low }}$ and $\alpha_{\text {high }}$, with high values for flat angles and low values for acute angles (Fig. 4a). Then, the weight $\alpha(s)$ is again defined by the profile function $f_{p}^{\left(s_{0}, \delta\right)}(s)$ in order to modulate the regularization constraints according to the distance to the bifurcation:

$$
\alpha(s)=\alpha_{\min }+\left(\alpha_{\text {high }}-\alpha_{\min }\right) f_{p}^{\left(s_{0}, \delta\right)}(s)
$$

Fig. 4b shows the profile we finally obtain for $\alpha(s)$ for the three curves delineating the bifurcation in our example of Fig. $2 b$. The regularization is high for points far from the junction points, to get smooth curves, and lower around the bifurcation. The more acute the angle, the more relaxed the constraint: for example, $\alpha_{\min } \simeq 13$ for $V_{3}$ (blue curve) for which $\theta=84^{\circ}$ while $\alpha_{\text {min }} \simeq \alpha_{\text {high }}$ for $V_{1}$ (red curve) where $\theta=166^{\circ}$.
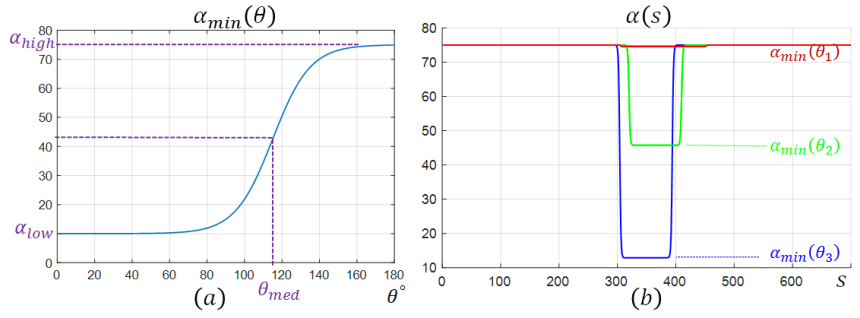

Fig. 4: Calculation of $\alpha(s)$. (a) Determination of $\alpha_{\min }$ as a function of the angle at the bifurcation. (b) Profiles $\alpha(s)$ for the three contours ( $s$ in pixels), parametrized by the abscissa $s_{0}$ of the junction point, the margin $\delta$ and the calculated minimum value $\alpha_{\min }$.

The energy functional $E\left(V_{i}\right)$ (Eq. 1) is classically minimized by solving numerically the associated Euler-Lagrange equation [11]. The active contour model is applied on the three curves $V_{i}$ independently. This last segmentation step leads to a precise and regular segmentation of the bifurcation (Fig. 5). Moreover, it respects the initial segmentation of the branches. The functions $\alpha$ and $\varphi$ have a suitable profile while being differentiable. There are five parameters to set: $\varphi_{0}, \alpha_{\text {low }}, \alpha_{\text {high }}, \theta_{\text {med }}$ and the factor $\gamma$ setting the slope of $\alpha_{\min }(\theta)$ (Eq. 5, Fig. 4a). We tuned them empirically on a subset of five images representative of the angles and diameters we have in our dataset, just by observing the behavior of the model. We also verified that the final values are not too sensitive, by checking that the final segmentation does not vary significantly if we shift slightly one parameter. Once these parameters are definitively fixed, the proposed method adapts itself to the geometry of the vessel branches, without any tuning. User's action is limited to the initialization step of the central reflections, an easy step which does not need to be precise. The next steps are fully automatic, which ensures a good reproducibility of segmentation results.

\section{EXPERIMENTS}

\subsection{Adaptive optics images and acquisition protocol}

To carry out this study, we have built an AO database of control, diabetic and CADASIL subjects, in order to make a statistical study of bifurcation morphometry in each pathology. The rtx 1 AOO camera from Imagine Eyes enables to visualize microscopic retinal structures with high resolution (pixel size equal to $0.8 \mu \mathrm{m}$, corresponding to a physical resolution of $1.6 \mu \mathrm{m} / \mathrm{pixel}$ ), non-invasively and without using contrast agents. Several images of the eye fundus were acquired, tracking one main artery emerging from the optic disk up to

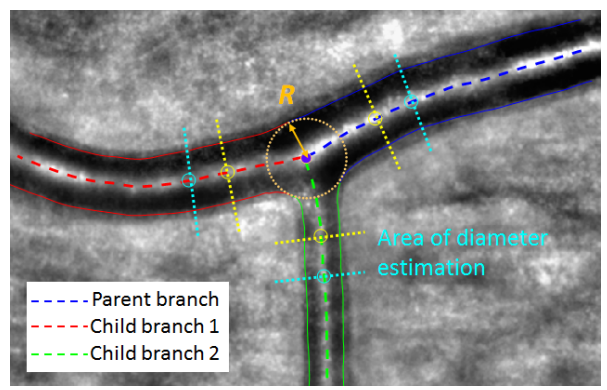

Fig. 5: Final segmentation and areas of diameter estimation.

the sixth bifurcation. Diameters of the acquired branches range from $90 \mu \mathrm{m}$ to $20 \mu \mathrm{m}$ for the smallest arterioles. Images were acquired from 23 control subjects, 28 patients with non-proliferative diabetes (NDR) and 25 patients with CADASIL syndrome. Each subject first underwent an ophthalmic examination, and visual control was done to ensure that the images were not blurred.

\subsection{Biomarker measurements}

Arterial bifurcation morphometry can be evaluated by measuring biomarkers derived from Murray's law [3]. The most known is the junction exponent $x$ defined by:

$$
d_{0}^{x}=d_{1}^{x}+d_{2}^{x}
$$

where $d_{0}$ is the parent diameter while $d_{1}$ and $d_{2}$ are the child diameters. Murray stated that $x=3$ for an ideal bifurcation. Clinical studies have shown a deviation of the junction exponent from this optimal value in peripheral arterial diseases [6], incident heart disease, stroke [4] and diabetes [5]. Nevertheless, solving Eq. 7 may lead to negative values of $x$, which has no physiological interpretation. This may happen in particular for pathological subjects [4]. For this reason, we have selected another biomarker, that is derived from the branching coefficient, defined by:

$$
\beta_{\text {measured }}=\frac{d_{1}^{2}+d_{2}^{2}}{d_{0}^{2}}=\frac{1+\lambda^{2}}{\left(1+\lambda^{x}\right)^{2 / x}}
$$

where $\lambda=d_{2} / d_{1}\left(d_{2}<d_{1}\right)$ measures the asymmetry of child branches. Considering an ideal bifurcation with an asymmetry coefficient $\lambda$, the optimal branching coefficient $\beta_{\text {optimal }}$ is given by the right hand side of Eq. 8 with $x=3$. Therefore, we calculate the deviation $\beta_{\text {dev }}$ to the optimal branching coefficient:

$$
\beta_{\text {dev }}=\beta_{\text {optimal }}-\beta_{\text {measured }}
$$

This biomarker is always calculable and provides information on the deviation to Murray's law optimum. In practice, we estimate the branch diameters in regions derived from the largest circle inscribed in the bifurcation (i.e. tangent to the segmentation), similarly to [12]. Let us denote by $R$ the radius of this circle. The measurement region starts at a distance equal to one radius $R$ from the intersection point between the circle and the central reflection, up to $2 R$ (see Fig. 5). We calculate the median of the diameters measured in this region (more robust to outliers than the mean value). Fig. 6 shows the biomarkers we obtained on our database.

For the results for $\beta_{d e v}$, we note a deviation in the positive direction of Murray's law which is consistent with [5] for the comparison between healthy and diabetic subjects. Yet, there is a trend toward a negative deviation for CADASIL subjects, which may indicate downstream increase of flow resistivity. 


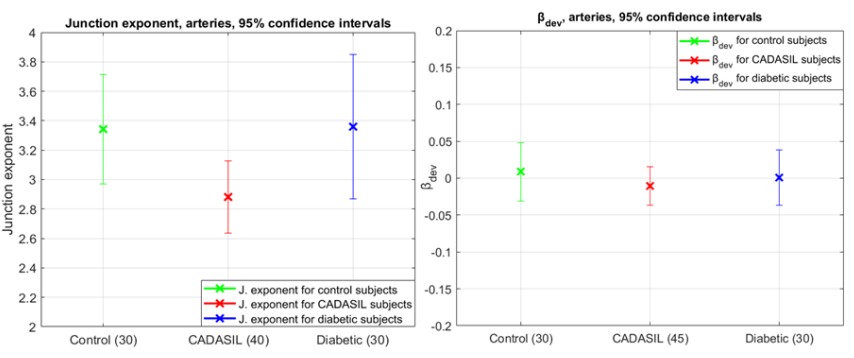

Fig. 6: Results for control subjects, diabetics and CADASIL at a 95\% confidence interval. Left: junction exponent $x$ (cases with $x>$ 0 only). Right: deviation $\beta_{\text {dev }}$ to the optimal branching coefficient.

\subsection{Quantitative evaluation}

Ten images (not trained) were selected from the database for the quantitative evaluation. This selection was done by the medical experts to ensure representativeness in terms of image quality and morphology variability encountered in clinical routine. Three physicians $\left(P h y s_{j}\right)$ segmented manually the set of images. Five images were processed twice by each physician to study the intra-expert variability. Let us denote by $V_{i}^{(S e g)}$ and $V_{i}^{(R e f)}$ two distinct segmentations of the contour $V_{i}$, where $V_{i}^{(R e f)}$ is chosen as a reference and $V_{i}^{(S e g)}$ as a segmentation to evaluate. The segmentation accuracy is given by the mean squared error (MSE) between the curves $V_{i}^{(R e f)}$ and $V_{i}^{(S e g)}$, calculated on a circular region centered on the bifurcation and of radius $4 R$. Then, we estimated the median diameters $d$ as described above, for each branch, in the segmentation and in the reference and, finally, the derived biomarkers. We denote by $\delta d_{0,1,2}, \delta \beta_{\text {dev }}$ and $\delta x$ the measured differences (averaged for the three branches for the diameters). The results are then averaged over the test cases to obtain mean and standard deviation values. Table 1 shows the intra-expert variability measured from the five segmentations realized twice by each expert. The physician Phys 3 , who obtained the most stable results on the biomarkers, was chosen as reference for the inter-experts and software/expert variability study. Table 2 summarizes the results obtained on the 10 images. Examples are shown in Fig. 7.

\begin{tabular}{|l|c|c|c|c|}
\hline & MSE & $\delta d_{0,1,2}$ & $\delta \beta_{\text {dev }}$ & $\delta x$ \\
\hline Phys $_{1}$ & $2.43 \pm 0.90$ & $+0.84 \pm 2.22$ & $0.00 \pm 0.09$ & $-0.10 \pm 0.49$ \\
Phys $_{2}$ & $2.80 \pm 0.99$ & $-0.62 \pm 3.98$ & $0.00 \pm 0.11$ & $+0.41 \pm 1.24$ \\
Phys $_{3}$ & $2.04 \pm 0.96$ & $-1.18 \pm 2.09$ & $+0.01 \pm 0.02$ & $+0.07 \pm 0.11$ \\
\hline
\end{tabular}

Table 1: Intra-expert variability (MSE and diameters expressed in pixels).

\begin{tabular}{|c|c|c|c|c|}
\hline Seg/Ref & MSE & $\delta d_{0,1,2}$ & $\delta \beta_{\text {dev }}$ & $\delta x$ \\
\hline Phys $_{1} /$ Phys $_{3}$ & $2.65 \pm 1.48$ & $+0.06 \pm 4.51$ & $-0.04 \pm 0.07$ & $-0.44 \pm 1.20$ \\
Phys $_{2} /$ Phys $_{3}$ & $3.25 \pm 1.84$ & $+0.52 \pm 6.15$ & $0.00 \pm 0.18$ & $-0.40 \pm 2.24$ \\
Software $/$ Phys $_{3}$ & $3.22 \pm 1.21$ & $+2.78 \pm 2.95$ & $+0.02 \pm 0.06$ & $+0.11 \pm 0.38$ \\
\hline
\end{tabular}

Table 2: Inter-expert variability and software/expert variability. Values are expressed in pixels for MSE and diameters.

The proposed method leads to MSE values within the same range as the inter-experts variability and slightly higher than the intra-expert variability. This demonstrates the accuracy of the proposed segmentation method. Considering the diameters, estimation errors are consistent with the measured $M S E$ but we note a positive bias that reveals a small over-segmentation, however with a smaller standard deviation than for the inter-experts evaluation. Nevertheless, our automatic method reaches the best accuracy regarding the biomarkers, both in terms of mean error and standard deviation, similar to the intra-expert accuracy and better than the inter-experts accuracy. However these errors are quite significant regarding the targeted application (Fig. 6), especially for $\beta_{d e v}$. This biomarker is always calculable but maybe more sensitive to segmentation imprecision than the junction exponent. This needs to be further investigated through a statistical analysis of significance. Examples are illustrated in Fig. 7. In (b), the blur on the child branch 2 (red arrow) leads to an uncertainty on the border localization, which results in significant differences between the manual and automatic estimates of $d_{2}$ and of the biomarkers. This study shows the difficulty of estimating biomarkers characterizing arterial bifurcations, despite the high resolution of AOO. The main limitation is due to the blur in the images, when the bifurcation is not exactly in the focal plane, which leads to uncertainty in the delineation of the lumen, even for experts.

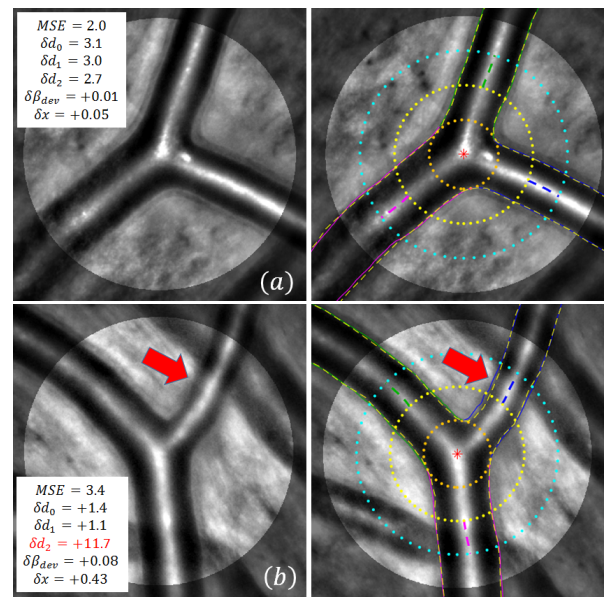

Fig. 7: Examples of segmentations and biomarker estimations. The manual segmentations are represented in yellow dashed curves.

\section{CONCLUSION}

We have proposed a method to segment arterial bifurcations in AOO, based on our previous approach for segmenting artery branches [8]. A new active contour model has been designed, to refine the segmentation at bifurcations. It is based on adaptive weighting of two regularization terms to cope with the specific geometry of every bifurcation and keep the initial segmentation where it is reliable. The quantitative evaluation has demonstrated a good accuracy of the proposed approach, with MSE values within the range of inter-experts variability. We have also shown promising preliminary results on biomarkers extracted from these segmentations to characterize pathologies. Future work aims at automatically evaluating the reliability of the diameter estimates, in order to discard biased biomarker values and better characterize CADASIL pathology. Moreover, it is now possible to consider the vascular tree as a whole, so to analyze blood circulation more globally.

Acknowledgments. The authors would like to thank Dr. V. Krivosic and Dr. C. Lavia of the ophthalmology department of Lariboisière hospital for providing AOO images, and the ophthalmologists of Quinze-Vingts Hospital for providing AOO images and for performing manual segmentations. 


\section{REFERENCES}

[1] H. Chabriat, A. Joutel, M. Dichgans, E. Tournier-Lasserve, and M.-G. Bousser, "Cadasil," The Lancet Neurology, vol. 8, no. 7, pp. 643-653, 2009.

[2] D'A. W. Thompson, On growth and form., Cambridge University Press, 1942.

[3] C. D. Murray, "The physiological principle of minimum work: I. the vascular system and the cost of blood volume," Proceedings of the National Academy of Sciences, vol. 12, no. 3, pp. 207-214, 1926.

[4] N. W. Witt, N. Chapman, S. A. McG. Thom, A. V. Stanton, K. H. Parker, and A. D. Hughes, "A novel measure to characterise optimality of diameter relationships at retinal vascular bifurcations," Artery Research, vol. 4, no. 3, pp. 75-80, 2010.

[5] T. Luo, T. J. Gast, T. J. Vermeer, and S. A. Burns, "Retinal vascular branching in healthy and diabetic subjects," Investigative Ophthalmology \& Visual Science, vol. 58, no. 5, pp. 2685-2694, 2017.

[6] N. Chapman, N. Witt, X. Gao, A.A. Bharath, A.V. Stanton, S.A. Thom, and A.D. Hughes, "Computer algorithms for the automated measurement of retinal arteriolar diameters," British Journal of Ophthalmology, vol. 85, no. 1, pp. 74-79, 2001.

[7] D. Lesage, E. D. Angelini, G. Funka-Lea, and I. Bloch, "A review of 3D vessel lumen segmentation techniques: Models, features and extraction schemes," Medical Image Analysis, vol. 13, pp. 819-845, 2009.

[8] N. Lermé, F. Rossant, I. Bloch, M. Paques, E. Koch, and J. Benesty, "A fully automatic method for segmenting retinal artery walls in adaptive optics images," Pattern Recognition Letters, vol. 72, pp. 72-81, 2016.

[9] S. Arichika, A. Uji, S. Ooto, Y. Muraoka, and N. Yoshimura, "Comparison of retinal vessel measurements using adaptive optics scanning laser ophthalmoscopy and optical coherence tomography," Japanese Journal of Ophthalmology, vol. 60, no. 3, pp. 166-171, 2016.

[10] F. Rossant, I. Bloch, I. Ghorbel, and M. Paques, "Parallel double snakes. Application to the segmentation of retinal layers in 2D-OCT for pathological subjects," Pattern Recognition, vol. 48, pp. 3857-3870, 2015.

[11] M. Kass, A. Witkin, and D. Terzopoulos, "Snakes: Active contour models," International Journal of Computer Vision, vol. 1, no. 4, pp. 321-331, 1988.

[12] S. Ramcharitar, Y. Onuma, J.-P. Aben, C. Consten, B. Weijers, M.-A. Morel, and P. W Serruys, "A novel dedicated quantitative coronary analysis methodology for bifurcation lesions.," EuroIntervention: Journal of EuroPCR in collaboration with the Working Group on Interventional Cardiology of the European Society of Cardiology, vol. 3, no. 5, pp. 553-557, 2008. 